\title{
INDOOR AND OUTDOOR MOBILE MAPPING SYSTEMS FOR ARCHITECTURAL SURVEYS
}

\author{
M. Campi ${ }^{1}$, A. di $\operatorname{Luggo}^{1}$, S. Monaco ${ }^{1}$, M. Siconolfi ${ }^{1}$, D. Palomba ${ }^{1}$ \\ ${ }^{1}$ Department of Architecture University of Naples Federico II \\ campi@unina.it, antonella.diluggo@unina.it, stefaniamonaco.90@alice.it, mirkosico@live.it, daniela.palomba@unina.it
}

\section{Commission II, WG II/3}

KEY WORDS: mobile mapping, image-based survey, GPS, data processing

\begin{abstract}
:
This paper presents the results of architectural surveys carried out with mobile mapping systems. The data acquired through different instruments for both indoor and outdoor surveying are analyzed and compared. The study sample shows what is required for an acquisition in a dynamic mode indicating the criteria for the creation of a georeferenced network for indoor spaces, as well as the operational processes concerning data capture, processing, and management. The differences between a dynamic and static scan have been evaluated, with a comparison being made with the aerial photogrammetric survey of the same sample.
\end{abstract}

\section{INTRODUCTION}

\subsection{Mobile Mapping Systems (MMS)}

In the last decades, the creation of three-dimensional models was very important in different applications, and technological development opened up new areas of experimentation in the architectural survey. The revolution caused by the laser scanner suddenly modified the approach to the procedures for data capture and, at the same time, the advantages deriving from the possibility to have a large amount of information, in a relatively short time and with high precision, allowed for a more widespread use of this technology. Moreover, in many cases procedures integrated with several different instruments were necessary to meet increasingly specific needs.

In this context, technologies concerning dynamic scans have to be examined, in particular, LIDAR (Light Detection And Ranging) technology for the survey of large outdoor surfaces. This technology includes an intense interaction of three components installed in a vehicle: a laser scanner, a satellite positioning system and an inertial navigation system. The limit of this technology is the need to continuously determine the position through GNSS networks.

In this framework, IMMS instrumentations (acronym of Indoor Mobile Mapping Systems) are a solution to collect data through kinematic platforms, in the absence of a GNSS signal. The fundamental issue of positioning is resolved through a method of Simultaneous Localization \& Mapping (SLAM), based on an inertial system in which accelerometers and gyroscopes provide measurements to mark the position of an object in relation to a starting point, orientation, and speed. This allows to calculate its trajectory by creating or updating a map in an unknown environment. According to Riisgard and Blas (2003), the standard SLAM process consists of the following passages: extraction of reference points, data association, estimate of state, updating of state and reference points.

In recent years, several tools based on the SLAM method have been tested. One of the most characteristic is a handheld system formed by a light laser scanner with a range up to 15-30 $\mathrm{m}$ and an inertial measurement unit MEMS (IMU), mounted on a spring mechanism thanks to which it oscillates allowing for an individual 2D laser scanner to rebuild a 3D scan. Although this instrument (Zeb1), developed by CSIRO, gives a lower level of detail in comparison to a traditional laser scanner (Sirmacek et al., 2016), it is particularly suitable for the survey of spaces with a limited access, such as the Tower of Pisa (F. Tecchia, 2013) where other systems would probably be inadequate.

Another device which is similar to a camera due to its size is Viametrics Mid, working as a 2D lidar, a camera, an AHRS and a tablet PC and allowing to map a building along its interior.

Other systems to acquire motion pictures, making it possible to overcome the typical obstacles of indoor spaces, are mounted on backpacks. Nevertheless, the operator's movements generate some mistakes and an attempt is being made to solve them (Lauterbach et al., 2015).

In addition to these instruments, the most interesting solution in IMMS is represented by mobile carriages upon which the different components improving its functioning are installed. Their main difference is their kind of laser scanner. For example, the models produced by Viametrics and NavVis mount three 2D laser scanners oriented on different levels, whereas the following document will show the procedures and analysis of a survey carried out with an IMMS tool of Trimble Geospatial having a single laser scanner.

\subsection{Study area}

The subject of this paper is the indoor mobile mapping system survey carried out in a building located at the University of Naples Federico II at Monte Sant'Angelo in Naples, projected 
on a variant of a 1972 planning scheme in a hilly area at the edge of Fuorigrotta district. It includes several buildings which follow natural curves, according to the terrain topography. The abovementioned building is on three floors, where the two underground spaces house the technical rooms, while the three above-ground floors contain offices, a large library, conference halls, two areas of classrooms and shops.

\subsection{Aims of the study}

The survey of this building is included in a wider research project carried out at the Department of Architecture of the University of Naples Federico II. It is aimed at documenting the whole complex (more than $240.000 \mathrm{~m}^{2}$ ) in order to obtain more complete and updated documentation, given that any available information is incomplete and fragmentary. During the survey of one of the buildings, it was therefore considered appropriate to test the indoor mobile mapping technology, using it not only for the indoor spaces, but also for the external façades and outdoor spaces. The data obtained, i.e. the processed point cloud, was compared both with the data obtained from a photogrammetric survey of the entire area carried out with a drone in a previous survey as well as with those obtained from surveys with a static laser scanner.

\subsection{Instrumentation}

A GPS Trimble R8 receiver was used to manage the geolocation data and a Trimble S5 total station to adjust the various station points.

The TIMMS (Trimble Indoor Mobile Mapping System) of Trimble-Applanix (Figure 1) was used to acquire scans and pictures. This system consists of a carriage upon which the different components are installed. Its top has 6 cameras, 5 with the horizontal axis and one with a vertical axis, each of them with a $2 \mathrm{MP}$ resolution. During the scan, the height of the photographic system can be adjusted, after a modification of the working procedures. This system is able to process images which can give RGB data to be associated with the cloud, as well as spherical images to create digital panoramas.

In addition to a display, its central part includes a 3D Faro class 1 laser scanner, characterized by a scan angle equal to 300 vertical degrees, which does not involve the instrument rotation to the vertical axis, but on the contrary, it works as a profilometer and acquires data during its movement. A key element of the entire apparatus is the inertial measurement system, located in an integral position with the laser scanner, which, together with the two odometers installed on the wheels, allows to continuously mark the precise position of the instrument.

The lower part of the carriage contains the heaviest components such as batteries, in order to keep a low center of gravity and minimise oscillations.

Finally, a system of handles allows to move the instrument easily during its use on the flat as well as raise and carry it where the height changes.

\section{SURVEY EXECUTION}

\subsection{Operating process}

This survey was divided into two sessions and carried out by two operators. The first sessions, lasting about 4 hours, consisted of determining a GPS point network, whereas during the second session, lasting about 5 hours, a dynamic scan of the spaces was carried out. In particular, $10.000 \mathrm{~m}^{2}$ were first scanned by obtaining a total cloud of about 626.049 .124 points.

The entire process consisted of the following phases:

1. Measurement of GPS external points (network A);

2. Measurement of internal points with total station (network B);

3. Connection network A and network B;

4. Assignment of GPS coordinates to the internal points;

5. Scan of the external surfaces;

6. Scan of the internal surfaces;

7. Data processing.

\subsection{Georeferenced network of control points}

The first phase was aimed at processing a mesh of waypoints supporting the scan data orientation and pictures which should later be acquired. The absence of a GPS signal in the indoor spaces made it necessary to set up two networks, a reference external network and an internal one (Figure 2).

The external network (network A) was defined along the building perimeter, where the choice of the points, placed at a distance of 10 to $30 \mathrm{~m}$, took into account the need of a homogeneous distribution aimed at giving a suitable covering of surfaces. The

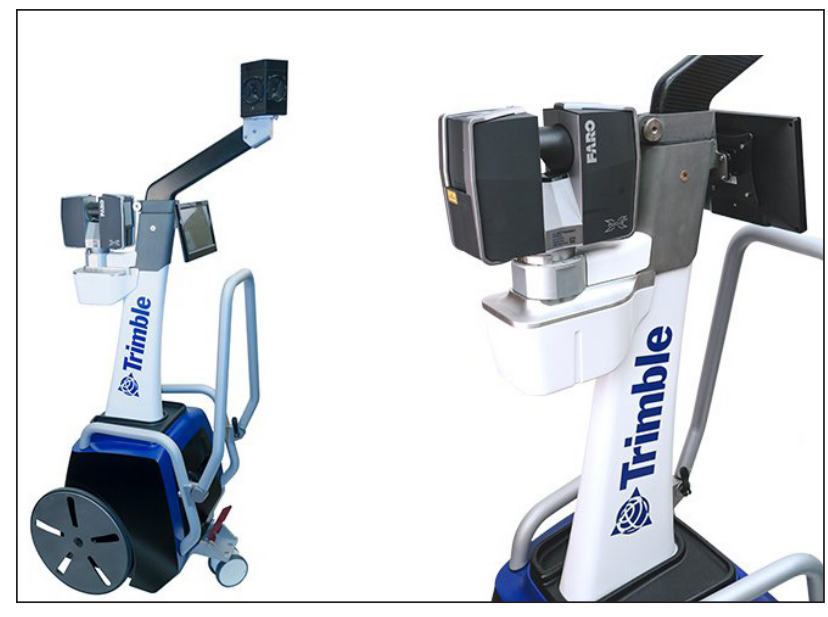

Figure 1. TIMMS (Trimble Indoor Mobile Mapping System)

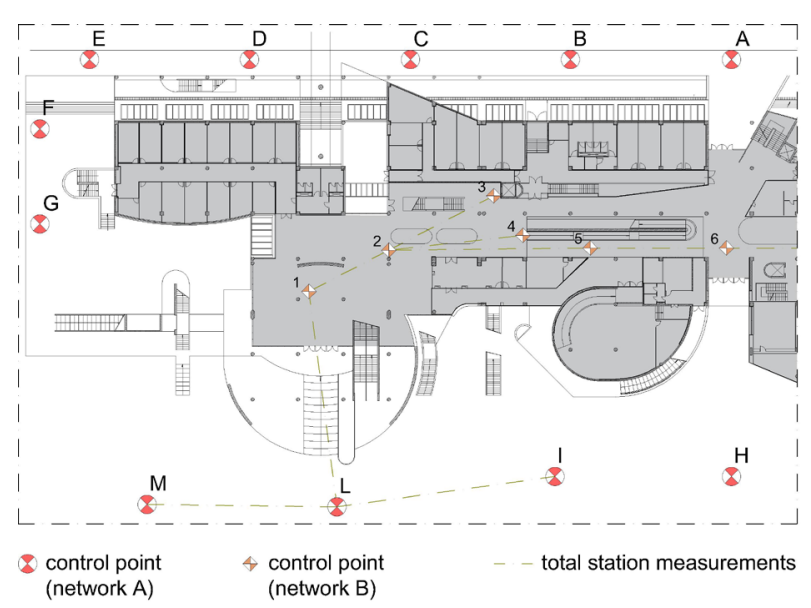

Figure 2. Network control points 

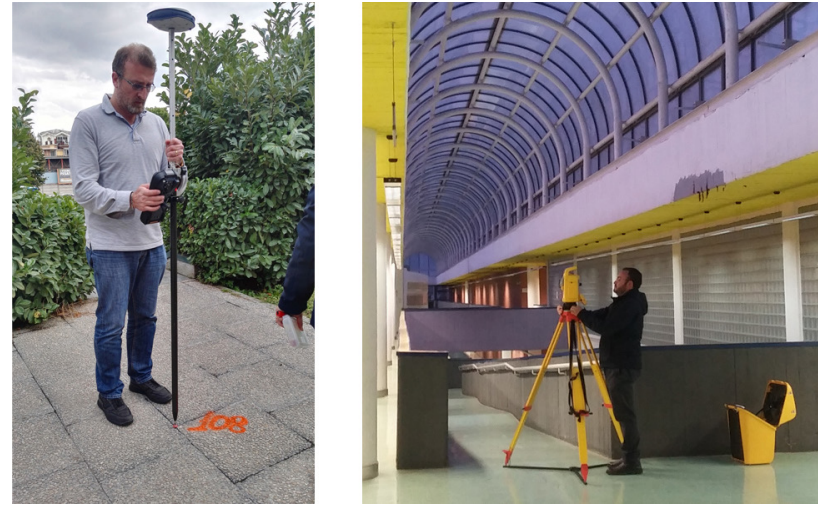

Figure 3. Measurements with GPS receiver and total station

GPS coordinates were then recorded for each individual knot (Figure 3). The accuracy in positioning is approximately $2 \mathrm{~cm}$. The internal network (network B) was defined so that the points, located on different levels, could be easily joined, prefiguring a three-dimensional network. Measurements were then carried out with the total station (Figure 3).

The two networks were linked through the connection between a point of the georeferenced external polygonal and one of the internal polygonals. In the following phase, the two networks were also associated by giving GPS coordinates to the internal points. This made it possible to create the file of the point mesh, which is helpful for scans.

\subsection{System initialization}

After the loading of the mesh GPS data into the computer, the system was initialized. The instrument was located near a selected point for the beginning of the scan (point A), identified thanks to a laser indicator, then the automatic calibration of the inertial device was started. This operation, lasting 20 minutes, was necessary to eliminate, or at least to reduce, any negative effects due to instability. The 20 minutes, divided into two sessions of 10 minutes, had some intervals thanks to a 180 degrees rotation of the device.

The data provided by the inertial device and by odometers determined the trajectory, which is fundamental for the connection of the point cloud and pictures.

\subsection{Data capture}

For the data capture, the instrument had to cover a sinusoidal trajectory passing through the waypoints with a stop of about 20 seconds near each of them. This performance facilitated data acquisition since it allowed the laser scanner to read more completely the depth of the spaces as well as reduce shadows, while also considering that the post-processing software maximizes the trajectory according to the morphological overlapping of the clouds.

In parallel to the scan, numerous spherical photos were taken with an interval of one meter.

The survey phase was fluid, with short interruptions due to the sudden height differences. In particular, near the stairs, inertial instrumentation was deactivated to be eventually reactivated after the device had been manually moved from one level to another. Once the process was completed, the project concerning the external surfaces was closed near the opening point (point A). The same procedure was then carried out indoors.

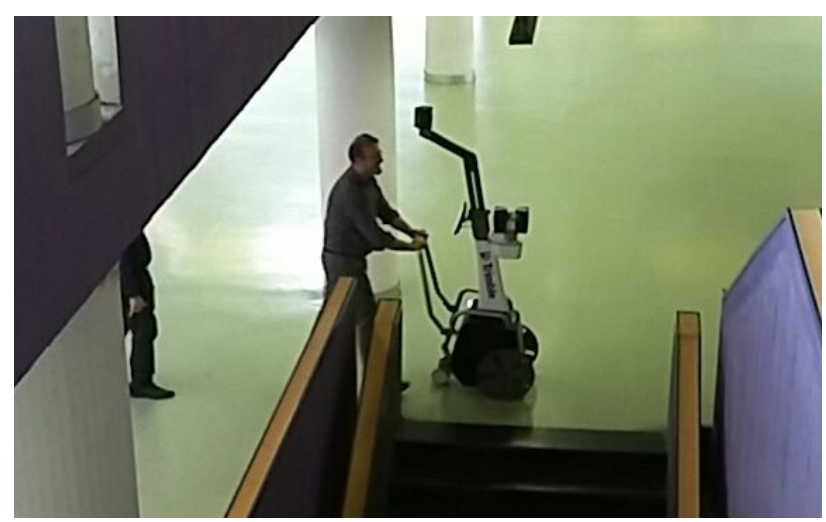

Figure 4. Indoor Scanning

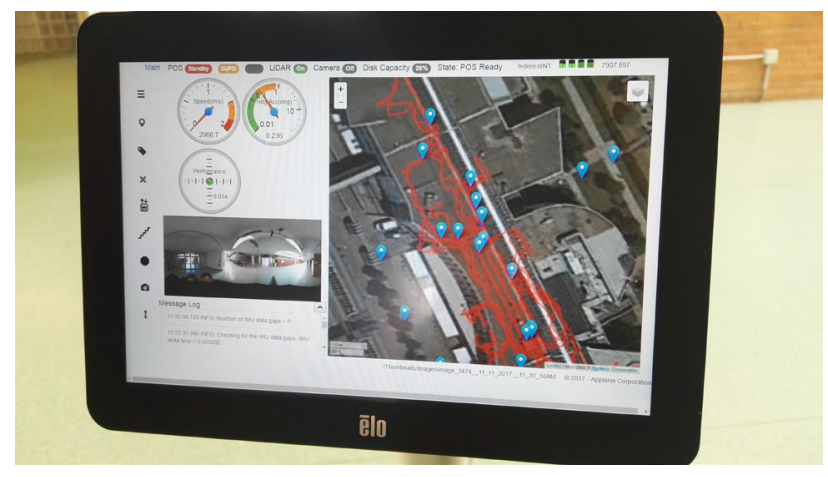

Figure 5. Instrumental interface

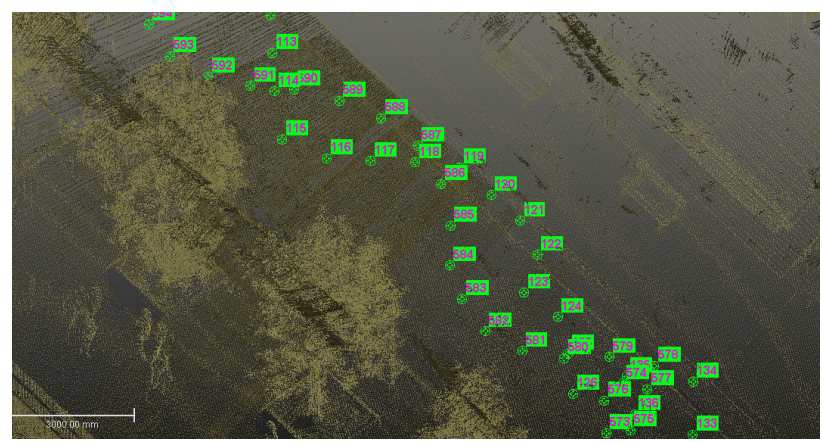

Figure 6. Position of photographic shots

\subsection{Data processing}

There are three kinds of data generated during acquisition obtained from the indoor and outdoor projects: point clouds (. $f s)$, spherical photos (.png) and position data from the points of support, the inertial system and the odometers (.pos).

These raw data were imported into the management software and processed so as to obtain files which can be used in .las format for the point clouds and in .jpg format for images. This work allowed to obtain clouds with a density of points varying from 1 to $5 \mathrm{~cm}$. 


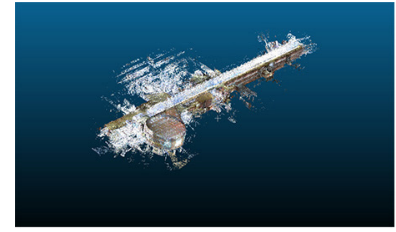

indoor point cloud

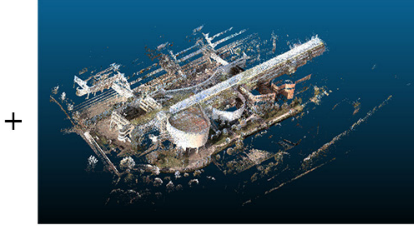

outdoor point cloud

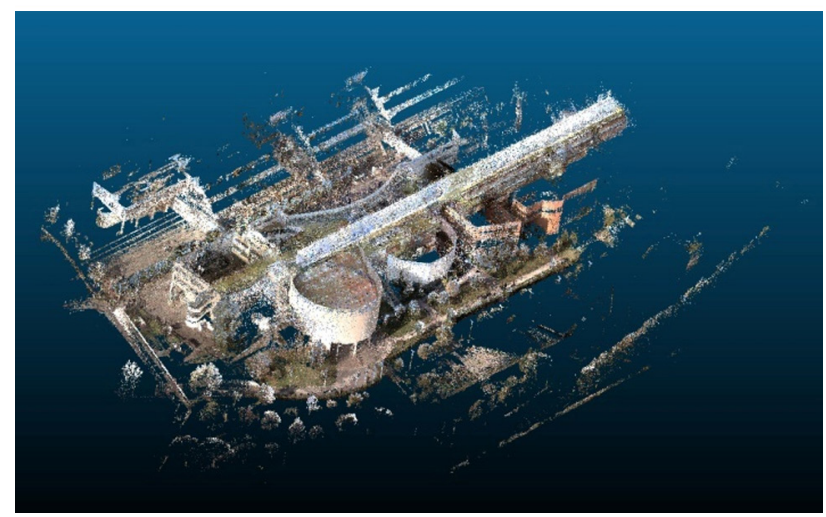

Figure 7. Fusion of point clouds

The clouds obtained with TIMMS were associated with a cloud of the whole complex previously acquired thanks to a photogrammetric survey by a drone. For this alignment, the homologous points between the two clouds had to be identified by overcoming some difficulties concerning the different density of the points, considering that the previous cloud was less bright, having the function only to document the external areas, coverings and the position of the various buildings.

The clouds obtained with the TIMMS were associated to a cloud of the entire complex previously acquired via a photogrammetric survey carried out with a drone (Figure 8). The instrument was a fixed-wing device (Ebee Sensefly), equipped with a Sony camera (compact digital) f. $4.5 \mathrm{~mm}$ at high resolution (24 MP) which performed a flight programmed through the Mission Planner software, at an altimetric range between $75 \mathrm{~m}$ and $115 \mathrm{~m}$ from ground level. The operation allowed to obtain 2330 frames with a value of GDS (Ground Sample Distance) equal to $1.5 \mathrm{~cm} /$ pix and with overlap and overside values that were around 80 and $60 \%$. The cloud, generated from the recognition of homologous points, was scaled and oriented with respect to a reference system obtained through GPS surveying.

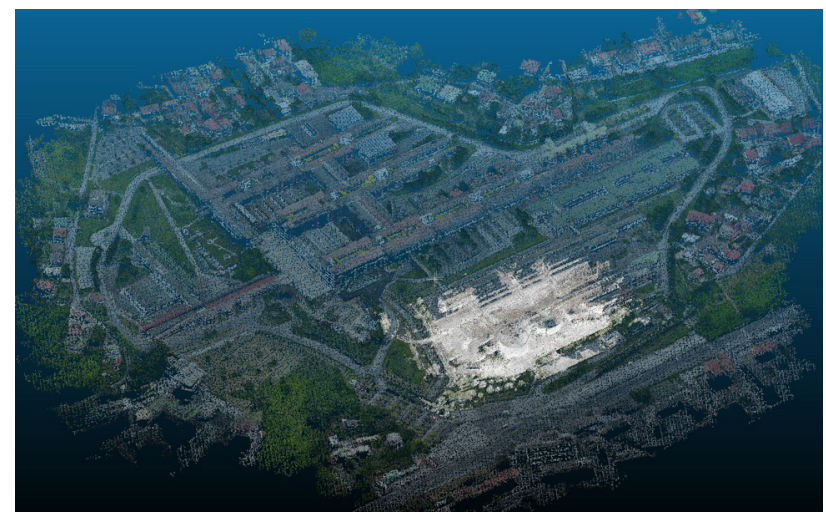

Figure 8. Matching point clouds obtained from TIMMS and UAV

\section{DATA MANAGEMENT}

Clouds in .las format were processed via an editing software such as Cloudcompare and Pointools, in order to carry out cleaning operations as well as obtain planimetric views, tables, sections, levels and all the information aimed at creating models such as the thickness of the walls, the structural grid, the inside partitions, along with the internal and external openings.

The management of large-scale data with averagely performing computers (quad-core, 16 GB RAM, discrete graphics card 4 GB) was highly complex so that the clouds had to be thin for a more rapid visualization with a partial loss of data near some of the details.

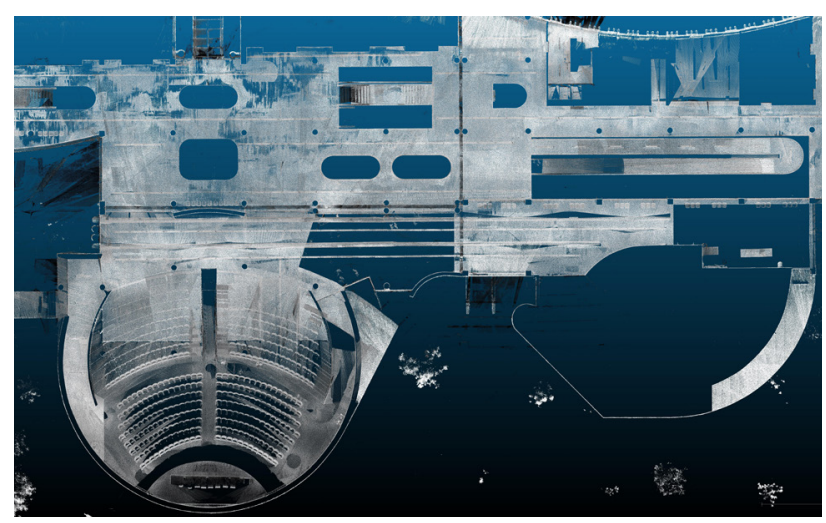

Figure 9. General plan
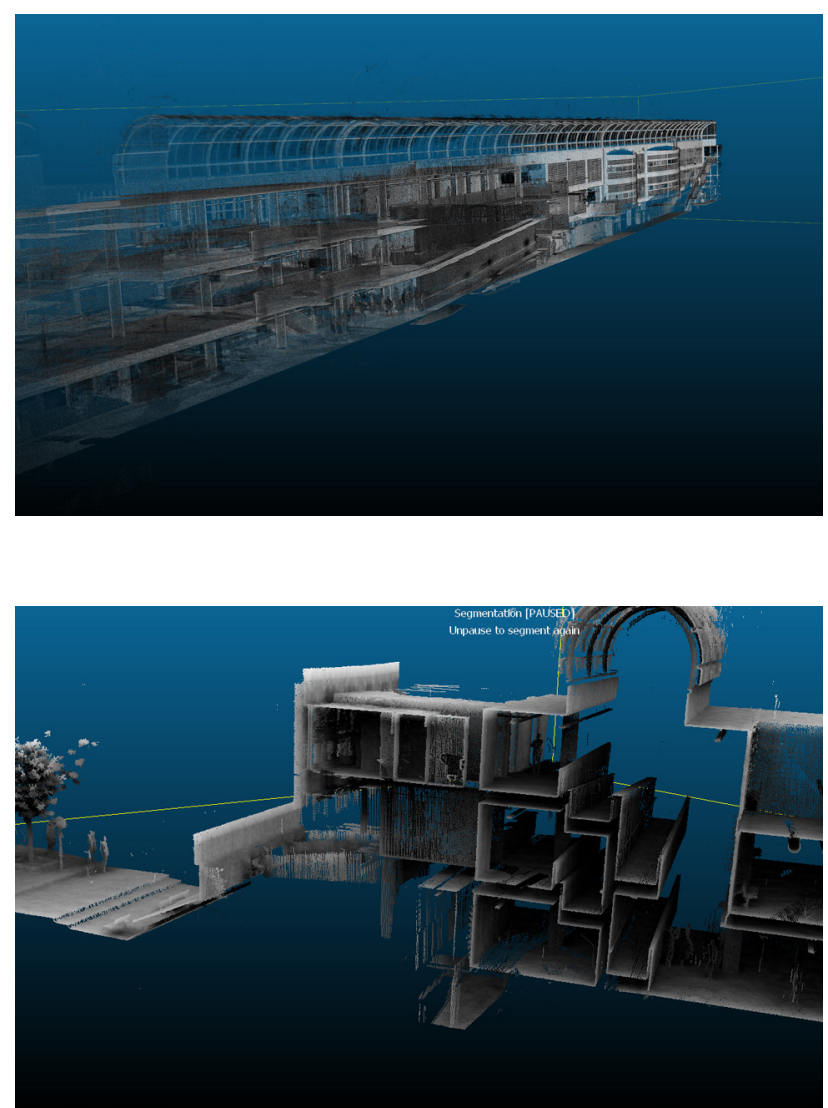

Figure 10 - 11. Cross sections 


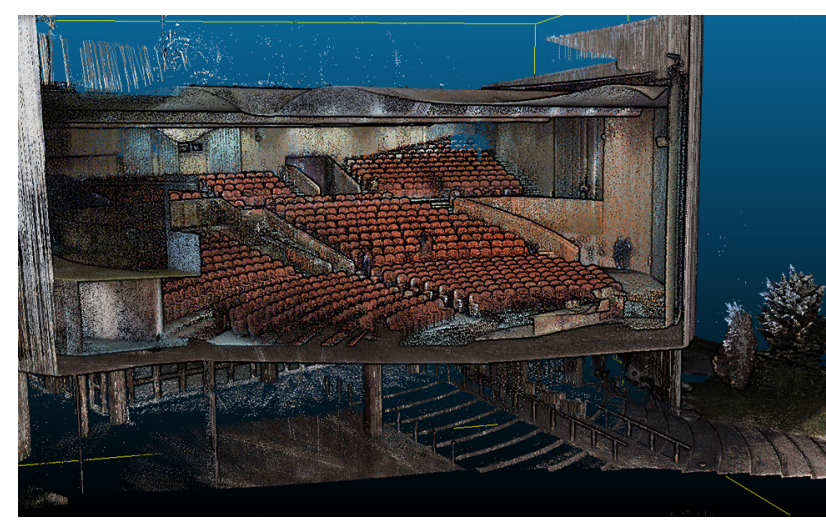

Figure 12. Cross section Congress Centre

\section{DIFFERENCES BETWEEN STATIC AND DYNAMIC SCANS}

As for a static laser scanner, one of the main negative factors of a dynamic scanner is either due to obstacles creating shadows or a variable illumination. The most important difference between a dynamic and a static device is a more rapid acquisition, an advantage in favor of MMS systems mainly due to a fluid and

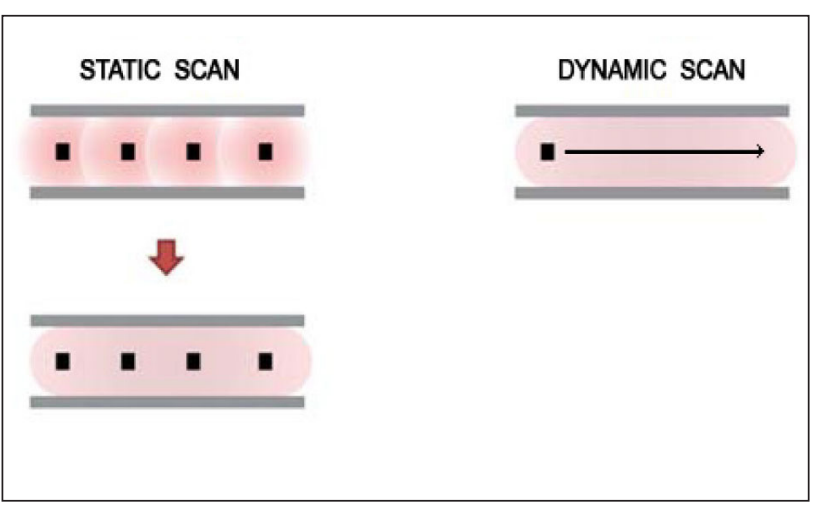

Figure 13. Differences between static laser scanner captures and TIMMS captures

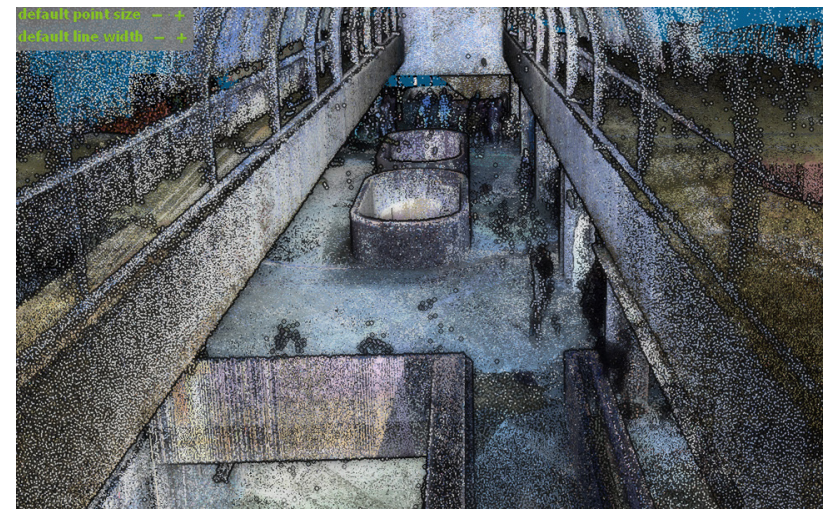

Figure 14. Point cloud RGB perspective continuous scan mode which does not need a new set-up for each station point or target (Figure 13)

Some case studies show that a dynamic system may reduce acquisition times by up to $80 \%$ or even more, as described in The American Surveyor (January 2017), with the survey of the terminals of Los Angeles airport, where it took 32 hours to acquire and process $55.000 \mathrm{~m}^{2}$ of surface in comparison with the 3 weeks of traditional laser scanner systems.

Time reduction also occurs during data processing, since the different scans must not be associated, as in the case of the static laser scanner, and this significantly decreases any installation errors of the components.

Nevertheless, it is worth noting how in some parts of the cloud of the building under study, as for example at the circular pillars, there is an estimated deviation of up to $2 \mathrm{~cm}$ of the circular profile. However, the actual contour of the pillar is identified by superimposing the most recurring points.

As described below, a survey with laser scanners was carried out. With this tool, only a small area was detected compared to that covered by the TIMMS, since the aim was to compare the average variance between the two types of acquisition. However, according to the working methods used, the temporal differences reported in the above-mentioned case studies can be considered plausible.

Another important aspect regards the acquisition of 360 degree photos for each meter. These images were used to obtain digital views, grouped in virtual tours created by specific software and displays. From these photos, TIMMS cannot obtain reliable RGB values for a correct return of a colored point cloud. In fact, there is a shift between the real point and the color value since the camera is set apart from the scanner, as well as because of the acquisition of spherical photos (Figures 14-15). From this point of view, both static and photogrammetric scans give a better graphic result.

Finally, a dynamic scan requires a certain amount of special attention in the speed of execution of the survey, since the scans may be more or less faded. The actual resolution of the cloud depends on the operator, since the maximum vertical scanning speed is $97 \mathrm{~Hz}$. Therefore, the device includes a digital speedometer allowing to control speed. There are other variations in the presence of slopes or irregular paving. The first case gives an inclined scan, the second a blurry scan (Figure 16).

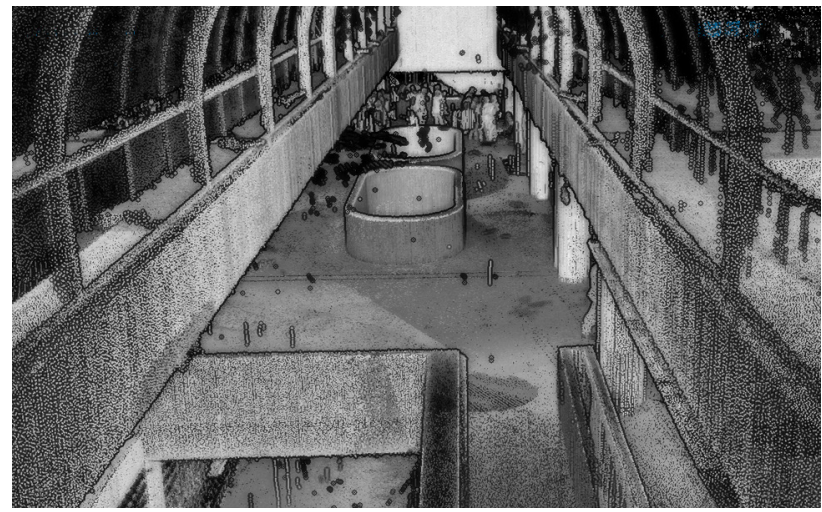

Figure 15. Reflectance perspective 


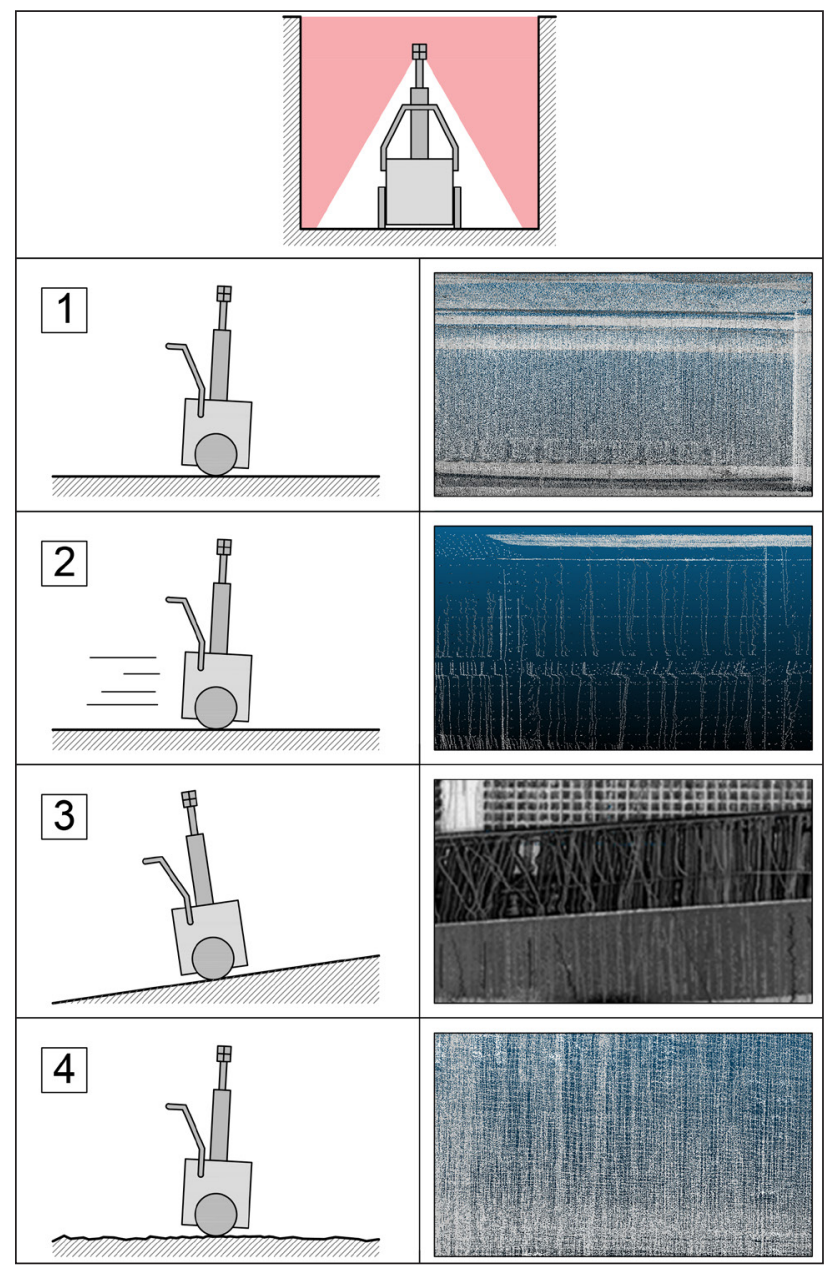

Figure 16. Data resolution by different conditions.

(1-regular pace; 2 - speedy pace; 3 -inclined plane; 4 irregular plane)

\section{COMPARISON OF POINT CLOUDS}

The analyzes compare the following data:

- DRONE photogrammetric cloud, realized to have a general reference of the entire university complex as well as to make considerations on a large scale (resolution 1 point every 5-10 $\mathrm{cm}$;

- TIMMS dynamic laser scanner cloud, carried out for the modeling of the indoor and outdoor elements of the building of the Common Centers (resolution 1 point every $2 \mathrm{~cm}$ );

- FARO static laser scanner cloud, used to make additional evaluations (resolution 1 point every $2 \mathrm{~cm}$ ).

\subsection{TIMMS/ drone outdoor cloud deviation}

The distance between the points was calculated with the Cloud Compare software. The result obtained is a mapping of the deviation between the two data: the average value is around 0,097 $\mathrm{m}$ as can be seen by the gaussian curve of the histogram in Figure 19. It is worth highlighting that the clearly different points were excluded, whose position is conditioned by different factors such as: objects (such as cars or people) not present in both clouds, shadow areas and gaps of the cloud obtained from the drone due to processing defects (reflective curvilinear surface of the congress center). The consideration of such data would have indicated a quantitatively significant error, but without value

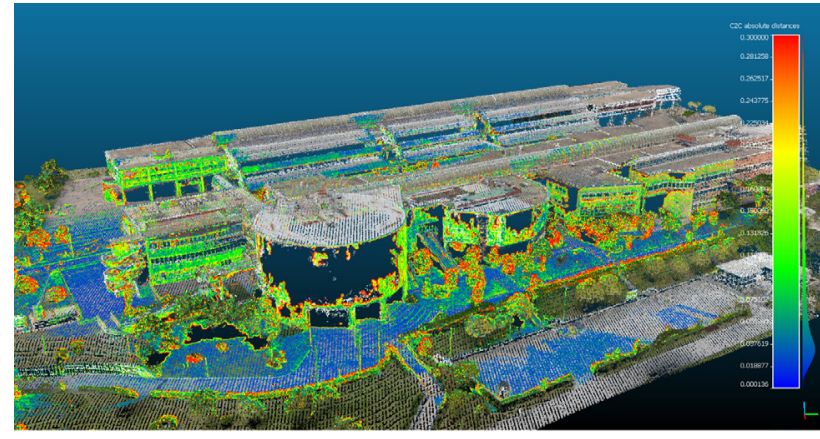

Figure 17. UAV/TIMMS main front view cloud deviation

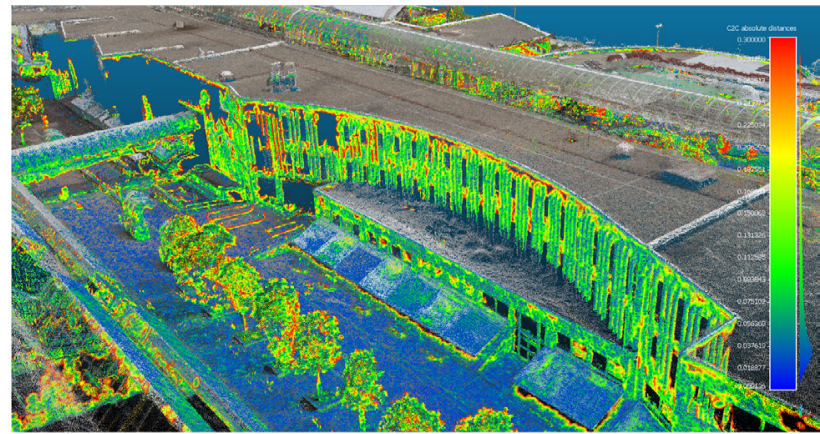

Figure 18. UAV/TIMMS main rear view cloud deviation

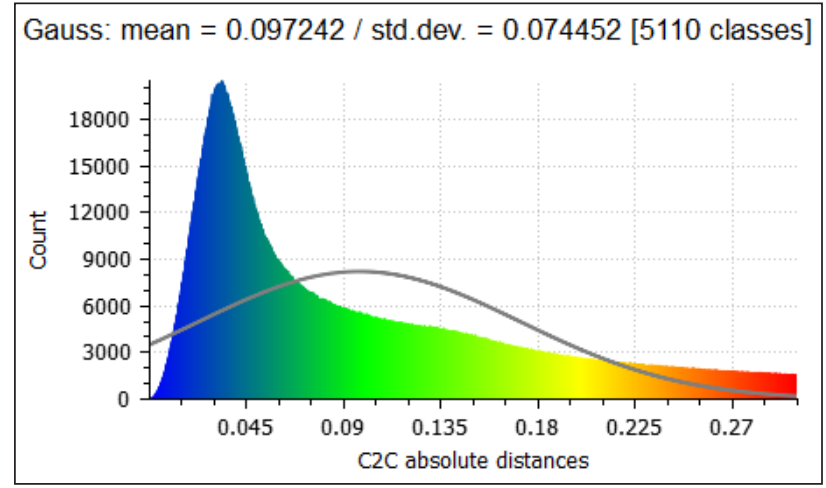

Figure 19. UAV/TIMMS histogram

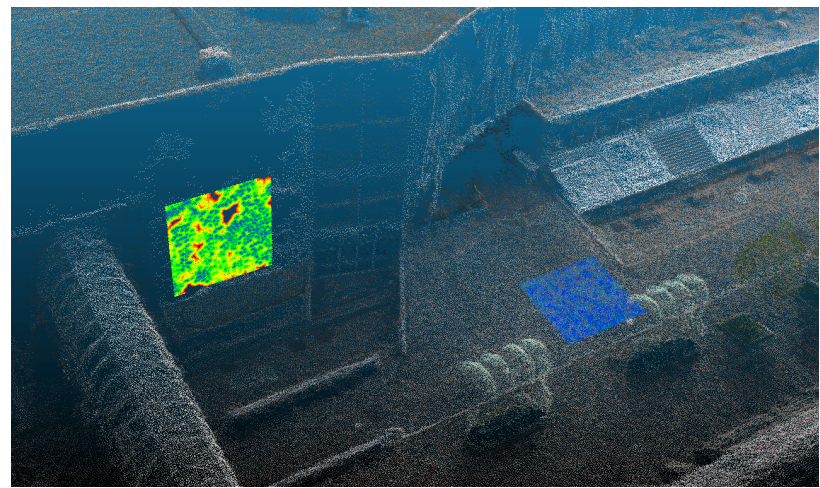

Figure 20. Horizontal and vertical surface extraction 


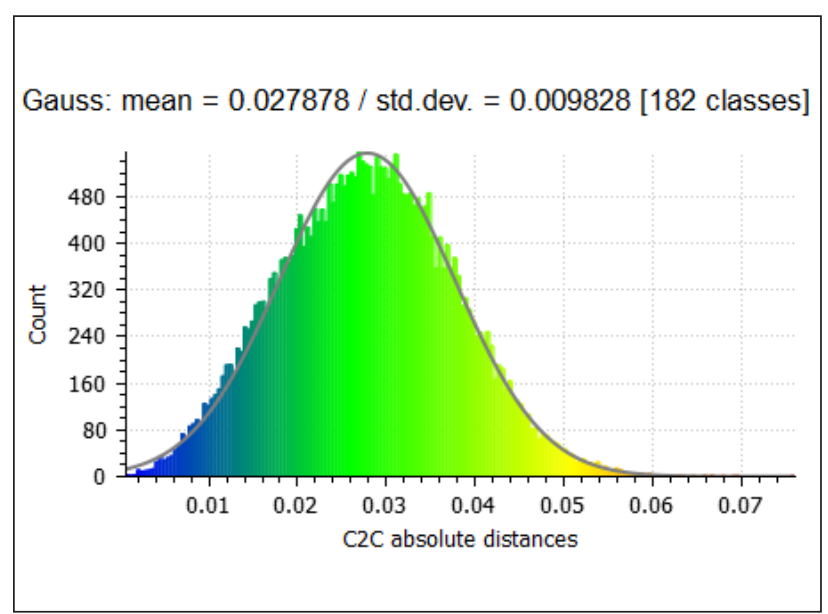

Figure 21. Horizontal plane histogram

\subsection{TIMMS/FARO cloud deviation}

The same comparative procedure was carried out between the point clouds obtained from the elaboration of the scans performed with laser scanner instrumentation in static mode (station points) and that from the laser scanner survey deriving from TIMMS instrumentation.

For the comparative operation, a limited area selected by both instruments was considered to be sufficient. The area identified is that of the ground floor corridor for indoor assessments and the area surrounding the rear entrance, for the outdoor comparison. The instrument used was a FARO Focus 3D s120 laser scanner, with which 13 scans were carried out at a distance of between 6 and 15 meters from each other. Moreover, in order to ensure a quick and more accurate alignment during registration, which took place via the Faro Scene software, 13 chessboard targets and 12 spherical targets were used.

By overlapping the two clouds, an average deviation of the points of about $1.5 \mathrm{~cm}$ was observed.

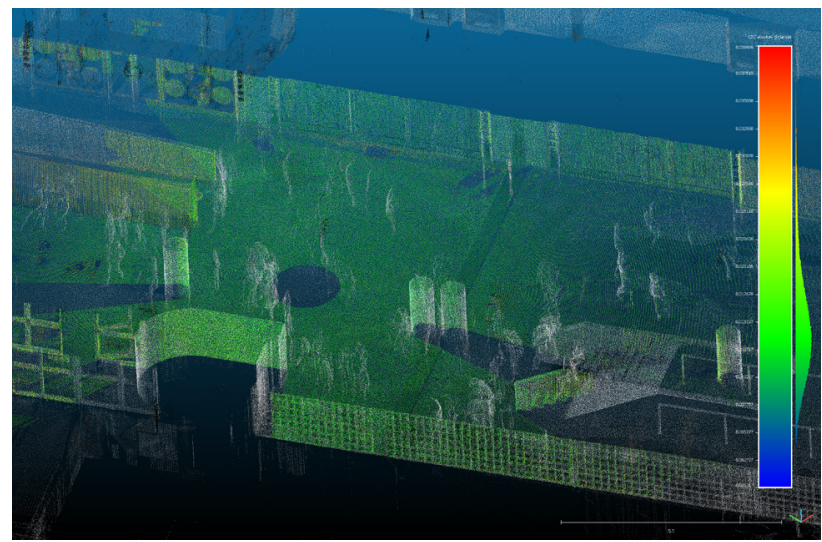

Figure 23. Elaboration of indoor TIMMS/FARO cloud deviation

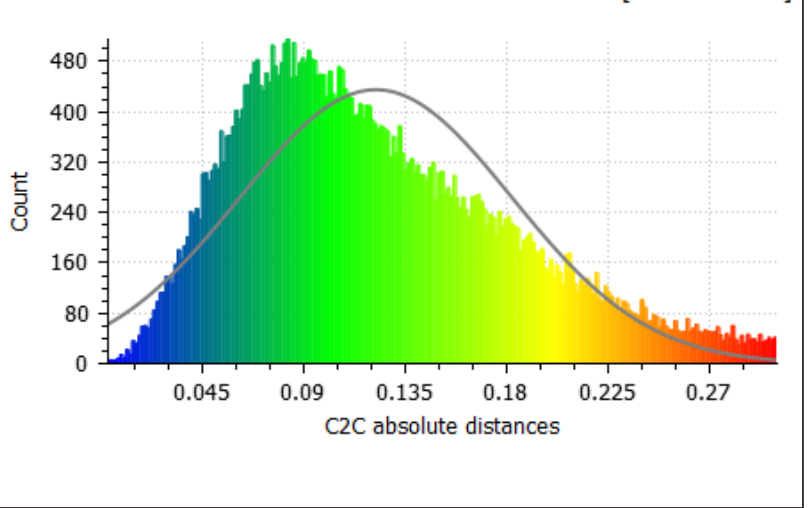

Figure 22. Vertical plane histogram

since there was no real comparison. It was therefore decided to consider only the data included in a range between 0,00 and $0,30 \mathrm{~m}$.

Observing the deviations in Figure 17 as well as in the histogram of Figure 19, it is possible to note that the points on the horizontal planes have a homogeneous and lower displacement with respect to those detected on the vertical planes. This condition is due to the fact that the horizontal plane corresponds to the photographic plane (obtained from the drone) and is therefore characterized by a greater degree of reliability.

For the two comparisons, sample surfaces of $4 \times 4 \mathrm{~m}$ were identified (Figure 20). The histogram of Figure 21 refers to the comparison of the data of the horizontal plane, with it being noted how the average deviation is $0,028 \mathrm{~m}$, while the histogram in Figure 22, referring to the sample of the vertical plane, shows an average displacement of $0,122 \mathrm{~m}$.

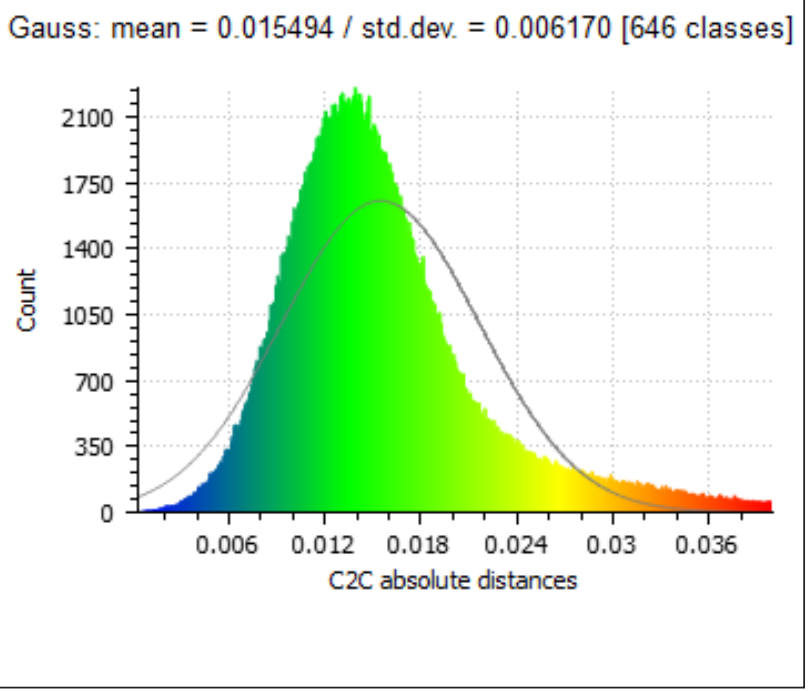

Figure 24. TIMMS/FARO histogram indoor 


\section{CONCLUSIONS}

One of the main aims of this work was to verify the behavior in the surveying of external spaces of an instrument designed to be used preferably indoors. The study sample covers approximately 150 linear meters, characterized by elevations articulated with different projections and curved walls.

The tested area is the left part of the abovementioned building, which includes the curved wall of the Congress Center and the external boulevard similar to a road.

As mentioned in the previous paragraphs, it is possible to deduce that the instrument realized outdoor surveys with the same accuracy of indoor surveying, while taking into account the difficulties due to the irregularity of the floor that could make the measurement of data slightly disturbed. However, this problem was solved by proceeding at a reduced speed.

It is possible to conclude that, given the reduced time for the execution of the survey, the acquisition speed of the instrument has allowed to obtain much more data than what could have been obtained with a static scanner at the same time.

Furthermore, the overall result obtained was useful to provide updated documentation of the building, in addition to using the cloud both to obtain two-dimensional information as well as be used in the construction of a BIM.

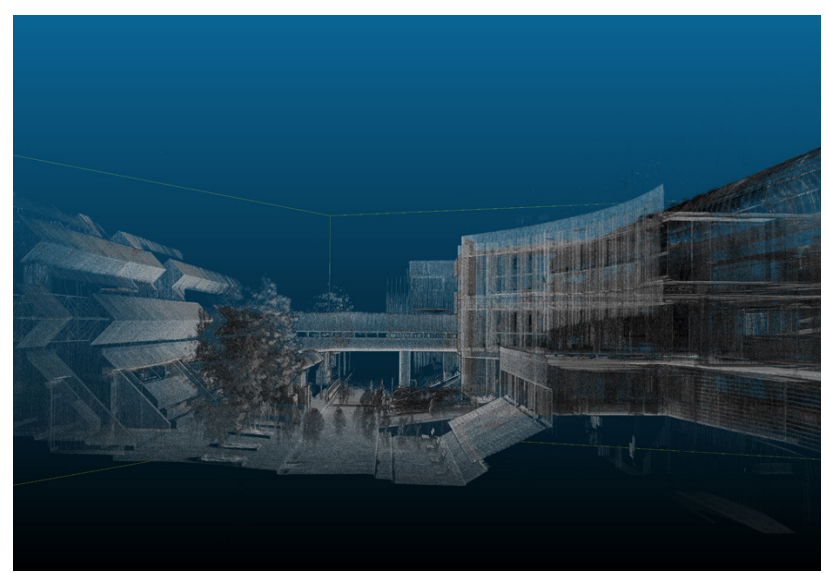

Figure 25. Outdoor TIMMS point cloud

\section{ACKNOWLEDGMENTS}

This paper is the result of research carried out by the Center of Research Urban/Eco, University of Naples Federico II (Italy). We would like to thank Trimble Company for making the tools necessary to carry out the research available as well as Donato Marcantonio and Michele Rubidio for supporting the research group during the survey and data processing phases.

\section{REFERENCES}

Bosse, M., Zlot, R., Flick, P., 2012. Zebedee: Design of a springmounted 3-D range sensor with application to mobile mapping. IEEE Trans. Robot. 2012, 28, 1104-1119.

Farella, E., Menna, F., Nocerino, E., Morabito, D., Remondino, F., Campi, M., 2016. Knowledge and valorization of historical sites through $3 d$ documentation and modeling, in ISPRS The International Archives of the Photogrammetry, Remote Sensing and Spatial Information Sciences. Volume XLI-B5, pp. 255-262.

Kukko, A., Kaartinen, H., Hyyppä, J., Chen, Y., 2012. Multiplatform mobile laser scanning: Usability and performance, in Sensors 2012, 12, 11712-11733.

Lauterbach, H. A., Borrmann, D., He $\beta$, R., Eck, D., Schilling, K., Nüchter, A., 2015. Evaluation of a Backpack-Mounted $3 d$ Mobile Scanning System, in Remote Sens. 2015, 7(10), 13753-13781; doi:10.3390/rs71013753.

Remondino, F., Nocerino, E., Toschi, I., Menna, F., 2017. A critical review of automated photogrammetric processing of large datasets, Int. Arch. Photogramm. Remote Sens. Spatial Inf. Sci., XLII-2/W5, 591-599, https://doi.org/10.5194/isprsarchives-XLII-2-W5-591-2017, 2017.

Riisgaard, S., Blas, M. R., 2003. SLAM for Dummies-A Tutorial Approach to Simultaneous Localization and Mapping.

Sirmacek, B., Shen, Y., Lindenbergh, R., Zlatanova, S., Diakite, A., 2016. Comparison of ZEB1 and LEICA C10 indoor laser scanning point clouds, in ISPRS Ann. Photogramm. Remote Sens. Spatial Inf. Sci., III-1, 143-149, https://doi.org/10.5194/ isprs-annals-III-1-143-2016, 2016.

Stenmark J., 2017. Takes Off at LAX, in The American SurveyorJanuary 2017.

Thomson, C., Apostolopoulos, G., Backes, D., Boehm, J., 2013. Mobile laser scanning for indoor modelling, in ISPRS Ann. Photogramm. Remote Sens. Spat. Inf. Sci. 2013, II-5/W2, 289293. 